\title{
A note on graphs without short even cycles
}

\author{
Thomas Lam * Jacques Verstraëte ${ }^{\dagger}$ \\ Submitted: Apr 6, 2004; Accepted: Mar 25, 2005; Published: Apr 6, 2005 \\ Mathematics Subject Classifications: 05C35, 05C38
}

\begin{abstract}
In this note, we show that any $n$-vertex graph without even cycles of length at most $2 k$ has at most $\frac{1}{2} n^{1+1 / k}+O(n)$ edges, and polarity graphs of generalized polygons show that this is asymptotically tight when $k \in\{2,3,5\}$.
\end{abstract}

\section{Introduction}

In this note, we study graphs without cycles of prescribed even lengths. For a finite or infinite set $\mathcal{C}$ of cycles, define $\operatorname{ex}(n, \mathcal{C})$ to be the maximum possible number of edges in an $n$-vertex graph which does not contain any of the cycles in $\mathcal{C}$. The asymptotic behaviour of the function $\operatorname{ex}(n, \mathcal{C})$ is particularly interesting when at least one of the cycles in $\mathcal{C}$ is of even length, and was initiated by Erdös [5]. In general, it is the lower bounds for $\operatorname{ex}(n, \mathcal{C})$ - that is, the construction of dense graphs without certain even cycles - which are hard to come by. The best known lower bounds are based on finite geometries, such as polarity graphs of generalized polygons [9], and the algebraic constructions given by Lazebnik, Ustimenko and Woldar [8] and Ramanujan graphs of Lubotsky, Phillips and Sarnak [11]; see also [10]. In the direction of upper bounds, the first major result is known as the even circuit theorem, due to Bondy and Simonovits [3], who proved that $\operatorname{ex}\left(n,\left\{C_{2 k}\right\}\right) \leq 100 k n^{1+\frac{1}{k}}$. A more extensive study of $\operatorname{ex}(n, \mathcal{C})$ was carried out by Erdős and Simonovits [6]. Our point of departure is the study of $\operatorname{ex}(n, \mathcal{C})$ when $\mathcal{C}$ consists only of the even cycles of length at most $2 k$. The main result of this article is the following:

Theorem 1 Let $k \geq 2$ be an integer. Then, for all $n$,

$$
\operatorname{ex}\left(n,\left\{C_{4}, C_{6}, \ldots, C_{2 k}\right\}\right) \leq \frac{1}{2} n^{1+\frac{1}{k}}+2^{k^{2}} n .
$$

Furthermore, when $k \in\{2,3,5\}$, the $n$-vertex polarity graphs of generalized $(k+1)$-gons in [9] have $\frac{1}{2} n^{1+1 / k}+O(n)$ edges and no even cycles of length at most $2 k$.

\footnotetext{
*Department of Mathematics, Massachusetts Institute of Technology, 77 Massachusetts Ave., Cambridge, MA 02139, USA. E-mail: thomasl@math.mit.edu

${ }^{\dagger}$ Faculty of Mathematics, University of Waterloo, 200 University Avenue West, Waterloo, Ontario, Canada. E-mail: jverstraete@math.uwaterloo.ca
} 
For the statement about the number of edges in the polarity graphs, see [9], page 9. Theorem 1 extends the Moore bound (see [2]) up to an additive term, and a more recent result of Alon, Hoory, and Linial [1], who proved that an $n$-vertex graph without cycles of length at most $2 k$ has at most $\frac{1}{2}\left(n^{1+1 / k}+n\right)$ edges (see Proposition 6$)$. In other words, we do not require that the odd cycles be forbidden, and the same bound still holds, but with a weaker additive linear term. Our result is also best possible in the following sense: if we forbid only the $2 k$-cycle in our graphs, then the upper bounds in Theorem 1 no longer hold - it was shown recently, in [7], that $\operatorname{ex}\left(n,\left\{C_{6}\right\}\right)>0.534 n^{4 / 3}$ and $\operatorname{ex}\left(n,\left\{C_{10}\right\}\right)>0.598 n^{6 / 5}$ as $n$ tends to infinity.

\section{Local Structure}

Let $G$ be a graph with no even cycles of length less than or equal to $2 k$. We write $P[u, v]$ to indicate that a path $P \subset G$ has end vertices $u$ and $v$, and we order the vertices of $P$ from $u$ to $v$. Let $\prec$ denote this ordering along $P$. A vine on a path $P$ is a graph consisting of the union of $P$ together with paths $Q\left[u_{i}, v_{i}\right]$ which are internally disjoint from $P$ for $i=1,2, \ldots, r$, and where $u \preceq u_{1} \prec v_{1} \preceq u_{2} \prec v_{2} \preceq \cdots \preceq u_{r} \prec v_{r} \preceq v$. A uv-path of shortest length is called a uv-geodesic. A $\theta$-graph consists of three internally disjoint paths with the same pair of endpoints.

Lemma 2 Any $\theta$-graph contains an even cycle.

Proof. If $P, Q$ and $R$ are the internally disjoint paths in the $\theta$-graph with the same pair of endpoints, then $|P \cup Q|+|Q \cup R|+|P \cup R|=2|P|+2|Q|+2|R|$, which is even. Therefore one of the cycles $P \cup Q, Q \cup R$ or $P \cup R$ must have even length.

Lemma 3 Let $P^{*}$ be a uv-geodesic of length at most $k$. Then the union $H$ of all uv-paths of length at most $k$ is a vine on $P^{*}$ and $P^{*}$ is the unique uv-geodesic.

Proof. Suppose, for a contradiction, that $H$ is not a vine on $P^{*}$. Let $x \prec v$ be a vertex of $P^{*}$ at a maximum distance from $u$ on $P^{*}$ such that the union of all $u x$-paths in $H$ is a vine on $P^{*}[u, x]$. By the maximality of $x$, there is a $u v$-path $P$ of length at most $k$ such that $x$ has degree three in $P \cup P^{*}$. If $P$ has minimum possible length, then $P[x, y] \cup P^{*}[x, y]$ is the only cycle in $P \cup P^{*}$ for some $y \succ x$ on $P^{*}$. By the maximality of $x$, the union of all $u y$-paths in $H$ is not a vine. Therefore there must be a $u v$-path $Q$ of length at most $k$ such that $Q \cup P \cup P^{*}$ is not a vine on $P^{*}$. If $Q$ has minimum possible length, then $P \cup Q$ and $P^{*} \cup Q$ each have exactly one cycle. It follows that there is a path $Q[w, z] \subset Q$ such that

$$
Q[u, x]=P^{*}[u, x] \text { and } Q[x, w] \cup Q[z, v] \subset P[x, v] \cup P^{*}[x, v]
$$

and $Q[w, z]$ is internally disjoint from $P \cup P^{*}$. Since $P \cup P^{*} \cup Q$ is not a vine, $w \in$ $P[x, y] \cup P^{*}[x, y]$ and $w \neq y$. If $z \in P^{*}[y, v]$, then $P^{*}[x, z] \cup P[x, z] \cup Q[w, z]$ is a $\theta$-graph (see Figure 1). 


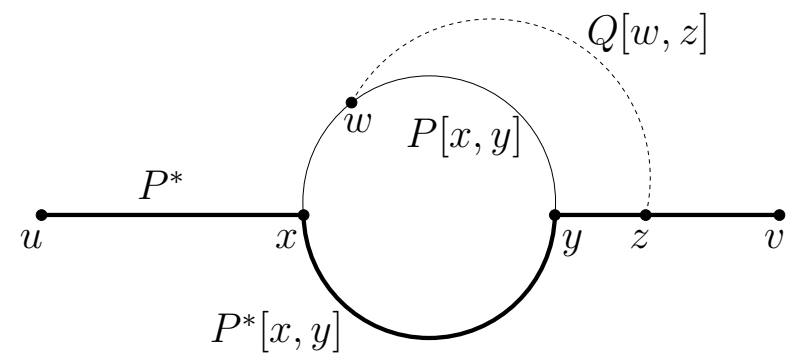

Figure 1: A $\theta$-graph in $Q \cup P \cup P^{*}$.

The cycles in this $\theta$ graph are $P[w, z] \cup Q[w, z] \subset P \cup Q$ and $P[x, y] \cup P^{*}[x, y] \subset P \cup P^{*}$ and $P^{*}[x, z] \cup Q[x, z] \subset P^{*} \cup Q$. Each of these cycles has length at most $2 k$, since the paths $P, Q$ and $P^{*}$ each have length at most $k$. By Lemma 2, one of these cycles has even length, which is a contradiction. A similar argument works when $z \notin P^{*}[y, v]$. Therefore $H$ is a vine on $P^{*}$.

To complete the proof, we must show that $P^{*}$ is the unique $u v$-geodesic. By definition, $H$ consists of the union of $P^{*}$ and paths $P_{i}=P_{i}\left[u_{i}, v_{i}\right]$ for $i \in[r]$, and let $P_{i}^{*}=P^{*}\left[u_{i}, v_{i}\right]$. Since each cycle $P_{i}^{*} \cup P_{i}$ is of length at most $2 k$, each cycle in the vine has odd length. Now suppose $P$ is another $u v$-geodesic. Then $P_{i} \subset P$ for some $i$. Since $P_{i} \cup P_{i}^{*}$ is an odd cycle, we may assume $\left|P_{i}\right|<\left|P_{i}^{*}\right|$. By replacing $P_{i}^{*}$ with $P_{i}$ on $P^{*}$, we obtain a $u v$-path of length $\left|P^{*}\right|-\left|P_{i}^{*}\right|+\left|P_{i}\right|<\left|P^{*}\right|$, which contradicts the fact that $P^{*}$ is a $u v$-geodesic. So $P^{*}$ is the unique $u v$-geodesic.

Henceforth, the paths in the vine on $P^{*}$ will be denoted $P_{i}=P_{i}\left[u_{i}, v_{i}\right]$, and $P^{*}\left[u_{i}, v_{i}\right]=P_{i}^{*}$, for $i \in[r]$. Let $\mathcal{P}_{k}(u, v)$ denote the set of all $u v$-paths of length $k$, and define the map

$$
f: \mathcal{P}_{k}(u, v) \rightarrow 2^{[r]} \quad \text { by } f(P)=\left\{i \in[r] \mid P_{i}\left[u_{i}, v_{i}\right] \subset P\right\} .
$$

Then $f(P)$ records the set of integers $i$ for which the path $P \in \mathcal{P}_{k}(u, v)$ uses the path $P_{i}\left[u_{i}, v_{i}\right]$ in the vine on $P^{*}$ instead of $P^{*}\left[u_{i}, v_{i}\right]$. Let $\mathcal{F}$ be the image of $\mathcal{P}_{k}(u, v)$ under $f$.

Lemma 4 The map $f$ is an injection, and the family $\mathcal{F}$ is an antichain of sets of size at most $k-\left|P^{*}\right|$ in the partially ordered set of all subsets of $[r]$.

Proof. By Lemma 3, each $P \in \mathcal{P}_{k}(u, v)$ is the union of some (possibly none) of the paths $P_{i}$ together with internally disjoint subpaths of $P^{*}$. Therefore the set $f(P)$ uniquely determines $P$, and $f$ is an injection. If two sets in $\mathcal{F}$ are comparable, say $f(P) \subset f(Q)$, then $|Q|>|P|$ and $Q \notin \mathcal{P}_{k}(u, v)$, which is a contradiction. So $\mathcal{F}$ is an antichain. Finally, any path $P \in \mathcal{P}_{k}(u, v)$ has length at least $\left|P^{*}\right|+|f(P)|$, by Lemma 3 , so all sets in $\mathcal{F}$ have size at most $k-\left|P^{*}\right|$. 
Theorem 5 Let $G$ be a graph containing no even cycles of length at most $2 k$. Then

$$
\left|\mathcal{P}_{k}(u, v)\right| \leq \max \left(\left(\begin{array}{c}
r \\
m
\end{array}\right): r \leq k \text { and } m=\min \left\{\left\lfloor\frac{r}{2}\right\rfloor, k-r\right\}\right) .
$$

The equality is achieved when $r=\left|P^{*}\right|$ and the vine on $P^{*}$ comprises $\left|P^{*}\right|$ triangles.

Proof. The family $\mathcal{F}$ is an antichain, by Lemma 4. By Sperner's Theorem and the LYM inequality [4], this means that $|\mathcal{F}| \leq\left(\begin{array}{c}r \\ m\end{array}\right)$ where $m=\min \left\{\left\lfloor\frac{r}{2}\right\rfloor, k-\left|P^{*}\right|\right\}$.

A non-returning walk of length $r$ in $G$ is a walk whose consecutive edges are distinct. Let $\mathcal{W}_{r}$ be the set of non-returning $r$-walks (for $r=0, \mathcal{W}_{0}$ consists of single vertices). The final result required for the proof of Theorem 1 is the following lower bound on the number of non-returning walks, by Alon, Hoory and Linial [1], which gives the best known upper bound on $\operatorname{ex}\left(n,\left\{C_{3}, C_{4}, \ldots, C_{2 k}\right\}\right)$ :

Proposition 6 Let $G$ be an n-vertex graph of average degree $d \geq 2$. Then $\left|\mathcal{W}_{r}\right| \geq$ $n d(d-1)^{r-1}$. Moreover, if $G$ has average degree $d \geq 2$ and no cycles of length at most $2 k$, then $d(d-1)^{k-1} \leq n$.

In [1], the number $\mathcal{W}_{r} / n d$ is denoted $N_{r-1}$ and shown to be less than $(d-1)^{r-1}$. The second statement of the Proposition is an immediate consequence of the main theorem there.

\section{Proof of Theorem 1}

Let $G$ be a counterexample to Theorem 1 with minimal number of vertices $n$ and average degree $d$. Then $d>n^{\frac{1}{k}}+2^{k^{2}}$, and $G$ has minimum degree at least $\lfloor d / 2\rfloor+1$, otherwise we remove a vertex of lower degree, keeping the average degree non-increasing, to obtain a smaller counterexample than $G$. We may also assume $n>2^{k^{2}}$. Now let $v$ be a vertex of $G$ of maximum degree, $\Delta$. Pick a breadth-first search tree $T$ rooted at $v$, and let $T_{r}$ be the set of vertices of $G$ at distance at most $r$ from $v$. Then no vertex of $T_{r}$ is joined to two vertices in $T_{r-1}$, and the set of edges in $T_{r-1} \backslash T_{r-2}$ form a matching, for all $r \leq k$. So every vertex of $T$ has degree at least $\delta-2$, where $\delta$ is the minimum degree in $G$, from which we deduce

$$
1+\Delta+\Delta(\delta-2)+\cdots+\Delta(\delta-2)^{k-1} \leq|V(T)| \leq n .
$$

Since $\delta>\lfloor d / 2\rfloor$ and $d>n^{\frac{1}{k}}+4$, we find $\Delta<2^{k-1} n^{\frac{1}{k}}$.

Now let $\mathcal{P}_{r}$ be the set of paths of length $r$ in $G$, and let $\mathcal{Q}_{r}=\mathcal{W}_{r}-\mathcal{P}_{r}$ be the set of non-returning walks with $r$ edges which are not paths. There are at least $\delta-k$ extensions of a given path of length $r$ in $G$, for any $r<k$. Therefore

$$
\left|\mathcal{P}_{k}\right| \geq(\delta-k)^{k-\ell}\left|\mathcal{P}_{\ell}\right| \quad \text { and } \quad\left|\mathcal{Q}_{k}\right| \leq \Delta^{k-1} k n<k 2^{(k-1)^{2}} n^{\frac{2 k-1}{k}} .
$$


By Lemma 3, for any pair $(u, v)$ of distinct vertices, joined by at least two paths of length $k$, there is a $u v$-geodesic of length $\ell<k$. By Theorem $5,\left|\mathcal{P}_{k}(u, v)\right|<2^{k}$, so the number of ordered pairs of vertices joined by exactly one $k$-path is at least

$$
\begin{aligned}
\left|\mathcal{P}_{k}\right|-2^{k} \sum_{\ell=1}^{k-1}\left|\mathcal{P}_{\ell}\right| & \geq\left|\mathcal{P}_{k}\right|\left(1-\frac{2^{k}}{\delta-k-1}\right) \\
& =\left(\left|\mathcal{W}_{k}\right|-\left|\mathcal{Q}_{k}\right|\right) \cdot\left(1-\frac{2^{k}}{\delta-k-1}\right) \\
& >\left(n d(d-1)^{k-1}-k 2^{(k-1)^{2}} n^{\frac{2 k-1}{k}}\right) \cdot\left(1-\frac{2^{k}}{\delta-k-1}\right) .
\end{aligned}
$$

In the last line, we used (1) and Proposition 6. There are $n(n-1)$ (ordered) pairs of distinct vertices which could be joined by a unique path of length $k$, so the expression above is less than $n^{2}$. Using $\delta-k-1 \geq \frac{d}{4}$ and substituting $d=n^{\frac{1}{k}}+2^{k^{2}}$ into the last line, we get

$$
\begin{aligned}
n^{2} & >\left(n\left(n^{\frac{1}{k}}+2^{k^{2}}\right)\left(n^{\frac{1}{k}}+2^{k^{2}}-1\right)^{k-1}-k 2^{(k-1)^{2}} n^{\frac{2 k-1}{k}}\right)\left(1-\frac{2^{k+2}}{n^{\frac{1}{k}}+2^{k^{2}}}\right) \\
& =\left(n^{\frac{2 k-1}{k}}\left(n^{\frac{1}{k}}+2^{k^{2}}\right)\left(1+n^{-\frac{1}{k}}\left(2^{k^{2}}-1\right)\right)^{k-1}-k 2^{(k-1)^{2}} n^{\frac{2 k-1}{k}}\right)\left(1-\frac{2^{k+2}}{n^{\frac{1}{k}}+2^{k^{2}}}\right) \\
& >\left(n^{\frac{2 k-1}{k}}\left(n^{\frac{1}{k}}+2^{k^{2}}\right)\left(1+n^{-\frac{1}{k}}(k-1)\left(2^{k^{2}}-1\right)\right)-k 2^{(k-1)^{2}} n^{\frac{2 k-1}{k}}\right)\left(1-\frac{2^{k+2}}{n^{\frac{1}{k}}+2^{k^{2}}}\right) \\
& >n^{2}\left(1+\frac{2^{k^{2}}}{n^{\frac{1}{k}}+2^{k^{2}}}\right)\left(1-\frac{2^{k+2}}{n^{\frac{1}{k}}+2^{k^{2}}}\right)>n^{2}
\end{aligned}
$$

which gives a contradiction. We must thus have $d<n^{\frac{1}{k}}+2^{k^{2}}$.

\section{Concluding Remarks}

If $G$ is $d$-regular, then picking a breadth first search tree as in the calculation of the maximum degree we obtain

$$
1+d+d(d-2)+\cdots+d(d-2)^{k-1} \leq n .
$$

So in this case we have $d<n^{\frac{1}{k}}+2$. The main points at which the large linear term is introduced in the proof of Theorem 1 is in the estimate of the maximum degree and the upper bound on $\left|\mathcal{Q}_{k}\right|$. We believe it should be possible to circumvent these bounds to obtain a linear term of the form $c n$, for some absolute constant $c$. Finally, we note that 
the analogous extremal problem when some of the short odd cycles are forbidden seems to be very difficult. For example, it is known that

$$
\frac{1}{2 \sqrt{2}} \leq \liminf _{n \rightarrow \infty} \frac{\operatorname{ex}\left(n,\left\{C_{3}, C_{4}\right\}\right)}{n^{3 / 2}} \leq \limsup _{n \rightarrow \infty} \frac{\operatorname{ex}\left(n,\left\{C_{3}, C_{4}\right\}\right)}{n^{3 / 2}} \leq \frac{1}{2}
$$

but the asymptotic value of $\operatorname{ex}\left(n,\left\{C_{3}, C_{4}\right\}\right)$ remains an open question (posed by Erdös).

Acknowledgements. The first author would like to thank Terence Tao for supervising him during his undergraduate thesis, which led to this work.

\section{References}

[1] N. Alon, S. Hoory, N. Linial, The Moore bound for irregular graphs, Graphs and Combinatorics 18 (2002), 53-57.

[2] N. Biggs, Algebraic Graph Theory, Cambridge University Press, Cambridge, second edition, 1993.

[3] J.A. Bondy, M. Simonovits, Cycles of even lengths in graphs, J. Combin. Theory Ser. B, 16, (1974) 97-105.

[4] K. Engel, Sperner theory, Encyclopedia of Mathematics and Its Applications 65, Cambridge University Press, Cambridge (1997).

[5] P. Erdős, Extremal problems in graph theory, 'Theory of Graphs and Its Applications' (M.Fiedler, Ed.), Academic Press, New York, 1965.

[6] P. Erdős, M. Simonovits, Compactness results in extremal graph theory, Combinatorica, 2(3) (1982), 275-288.

[7] Z. Füredi, A. Naor, J. Verstraëte, On the Turán number for the hexagon, preprint (2004).

[8] F. Lazebnik, V.A. Ustimenko and A.J. Woldar, A new series of dense graphs of high girth, Bull. Amer. Math. Soc. 32 (1995), 73-79.

[9] F. Lazebnik, V. A. Ustimenko and A. J. Woldar, Properties of Certain Families of 2k-Cycle Free Graphs, J. Combin. Theory Ser. B. 60, (1994), no. 2, 293-298.

[10] F. Lazebnik, V. A. Ustimenko and A. J. Woldar, Polarities and 2k-cycle-free graphs, Discrete Mathematics, 197/198, (1999), 503-513.

[11] A. Lubotzky, R. Phillips, P. Sarnak, Ramanujan graphs, Combinatorica 8 (1988), no. $3,261-277$. 